\title{
A hybrid approach to experimental control
}

\author{
Peter Dixon \\ University of Alberta, Edmonton, Alberta, Canada
}

\begin{abstract}
In the present article, I describe a new, hybrid approach to experimental control that combines the best features of general-purpose programs and specific-experiment programs. First, experimental control objects are developed in an object-oriented programming environment. Then, an XML file is used to select and arrange those control objects in order to carry out an experiment. A wide range of experiments can be implemented by specifying the arrangement of the control objects in the XML file, while, at the same time, the set of control objects can be easily extended for more demanding applications. An overview of a system using this approach is provided, along with some examples.
\end{abstract}

Psychologists in large numbers have been using computers to run experiments for 20 to 30 years. This means that psychologists (or their students or their research assistants) spend a great deal of time not running experiments, but getting their computers to run experiments. Moreover, a common constraint in how much and what research gets accomplished is what one can get a computer to do. One can track the concern and preoccupation with this activity by examining programs for the Society for Computers in Psychology over the years: Programs and packages designed to run experiments of various kinds have been a recurring theme that shows no sign of diminishing. There are two kinds of issues central to these articles: efficiency (i.e., how does one get computers to do what is needed quickly and with little effort?) and flexibility (i.e., how can one extend what can be done with computers to new paradigms, situations, and hardware?). Despite the continuing attention to these questions, they have not gone away. It would seem that although the field has come a long way in experimental control from merely replacing the tachistoscope, there are always new things that one might want to do effectively and efficiently with computers.

\section{Approaches to the Efficiency and Flexibility Goals}

Two broad classes of approaches to addressing these two goals of efficiency and flexibility exist. On one hand, one may develop a monolithic, general-purpose program that is intended to do everything one might wish to do in experimental control. This approach is exemplified by programs such as E-Prime (Psychology Software Tools Incorporated, n.d.), PsyScope (Bonatti, 2008), and SuperLab (Cedrus Corporation, 2008). Because such programs can do so many different things, one needs to write a fairly detailed script to run any particular experiment. At the other extreme are programs designed to run a particular experiment or perhaps a particular paradigm. If one is going to write such a program very many times, one typically develops a library of subroutines or collections of example code that one can piece together to construct new programs in any given instance.

General-purpose programs. A main advantage of the general-purpose program approach is efficiency: It is relatively easy to write a script to run a new experiment. Ideally, with such a program, one might be able to ask a well-motivated student, armed with only a tutorial and manual, to produce a working experiment in a week or two. However, there are also disadvantages to the approach. The main one is a lack of flexibility: It can sometimes be awkward or difficult to attempt something that has not been specifically anticipated by the program designers. For example, it is quite possible to have specialized hardware in one's lab that will not work with such a program. Or, conversely, one may have to limit the configuration of one's lab to what the software is known to be able to handle. In either event, the reliance on such a generalpurpose program may constrain the type of research one can carry out.

There are also other, more subtle, disadvantages. For example, general-purpose programs are expensive to develop, and few researchers do it on their own. Instead, such programs are commonly purchased from commercial vendors. The result is that if one finds a problem or limitation with a program, one generally cannot fix the problem on one's own, but must instead rely on the vendor to provide an update to the program. Finally, if the program is quite general and powerful, scripting a given experiment may be difficult or nonintuitive. This is particularly true for text-based scripting environments that have a great deal of expressive power. In effect, the complexity of the scripting environment may approximate that of a generalpurpose programming language.

Specific-experiment programs. A program specifically designed for a particular experiment is appropriate 
if one needs to address particular lab requirements or if one has a new paradigm that is not readily handled by a general-purpose program. Indeed, writing a program from scratch may sometimes be the only means of obtaining the needed flexibility when moving into new domains and methods. However, specific-experiment programs have drawbacks, too, the chief one being that the development of such programs can be a great deal of work. Moreover, the amount of effort is often prohibitive if one just wants to get a single experiment to run. Indeed, many people would probably abandon the use of computers if they had to develop a new program every time they wanted to collect data. Another disadvantage is that developing specificexperiment programs requires programming skills. Many psychologists (and probably most students) do not have those skills, and the programming would have to be contracted out. Thus, the cost of developing a new program may make it infeasible in many instances.

Somewhat-general programs. A compromise between the extremes of general-purpose programs and specific-experiment programs is to develop what might be termed "somewhat-general programs." Such programs can be used to carry out more than a single experiment or paradigm by changing the values of a few parameters but have no pretensions of being a general-purpose system. A good example is the ACTUATE system (Westbury, 2007), which runs a variety of paradigms associated with a clinical assessment battery. Such programs can be easy to use, since there is no complex scripting language to learn. Although they require significantly more work to develop than a specific-experiment program, the work may not be prohibitively expensive. If the research needs are not overwhelmingly diverse, this approach can be effective. However, by their very nature such programs have limited generality.

Object-oriented programming. Another solution to the efficiency/flexibility problem is object-oriented programming. In 1991, I argued that object-oriented programming would allow one to develop specific-experiment programs much more efficiently than could be done using the procedurally based approaches common at the time (Dixon, 1991). In this approach, one develops program objects that mirror the conceptual structure of an experiment; then, new paradigms are developed by programming variations on those objects. (It is interesting to note that PsyScope, which I would classify as a general-purpose program, uses a scripting language that is also objectoriented [Flatt, Cohen, \& Provost, 1994].)

The "object" in object-oriented programming consists of the data and routines that together accomplish a given task. Thus, an object encapsulates some well-specified functionality. A central feature of object-oriented programming is inheritance: One can define child objects that inherit the behavior of the parent object but add new functionality or carry out the old functions in new ways. When inheritance is applied to the problem of experimental control, one can map the programming objects onto the conceptual elements of an experiment. For example, one might have an object that runs an experimental trial in which a stimulus is presented and a response is col- lected; a child object might be defined to run a reaction time trial in which the response latency is also recorded; a further descendent might be defined for a priming trial in which the stimulus presentation consists of first presenting a prime and then presenting the target. In each case, the child object overrides the behavior of its parent to perform a related but new paradigm.

The advantage of object inheritance in this context is that new paradigms can be developed quickly by programming variations on older paradigms. Moreover, the variations are not ad hoc but, rather, reflect the development and conceptual basis of the new paradigm. Despite these advantages, there are some problems with object-oriented programming. To begin with, the effort needed to develop a program for an experiment remains relatively high, despite the reuse of code that comes with the approach. As a consequence, there is a tendency to write multipurpose programs that rely on parameters or input files to run a particular experiment. In other words, one may move in the direction of a somewhat-general program. The creation of many related object-oriented experimental control programs also tends to generate version problems. For example, if one has six different descendents of an RTText experiment, it may be unclear which should be used for the particular rapid serial visual presentation paradigm that one is trying to create. However, in my experience, the largest problem with the object-oriented approach is that students rarely have the requisite programming skills. As a consequence, the creation of experimental control programs can remain a bottleneck in getting research done in the lab.

\section{Scripting an Object-Oriented Program}

In the present article, I describe a new solution to the efficiency/flexibility problem that builds on the promise of object-oriented programming. The essence of this approach is to use XML as a format for describing the arrangement of program objects needed to conduct an experiment. This in effect generates a general-purpose program that is readily extensible. The approach reduces overhead in getting a single experiment to run, and students can acquire the relevant scripting skills with a minimum of difficulty. However, one still maintains the flexibility to develop new capacities and paradigms by adding new program objects as needed. I have developed a program using this approach, ESExpt, and it is in active use in my lab for a variety of paradigms, ranging from language production and numerical processing to psychological refractory period experiments, word-by-word reading paradigms, and movement trajectory monitoring (e.g., Dixon \& Glover, 2008; Dixon \& Li, 2007).

This approach uses the interesting (but not unique) ability of the Objective-C language to dynamically create an object from a textual representation of the object's class name. Consequently, one can describe the program needed to run an experiment with a text file that lists the objects used to carry out the experiment. With such a description, all that is needed is a program "stub" that reads the description file, dynamically creates the objects listed in the file, and then passes control to those objects. In effect, the file functions as a form of script that dictates 
Table 1

An XML Example

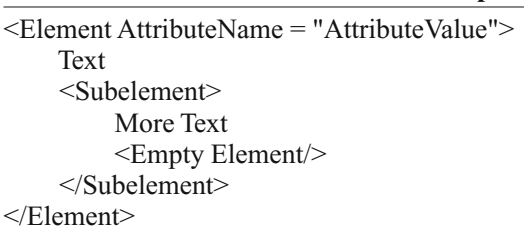

which objects to use and, by virtue of that, how the experiment will be carried out.

The other piece of this approach is to use XML as the format of the input text file. This approach is useful because the objects will have a hierarchical arrangement and it is easy to describe such a hierarchical structure using XML. In addition, the present implementation was done in the Cocoa environment in Mac OS X, and Cocoa comes with easy-to-use facilities for parsing XML files. Table 1 provides a brief illustration of the features of XML that are needed in the present context. (There are many sources for a more complete description of XML, e.g., Harold \& Means, 2004.) XML is similar to HTML but is somewhat more structured. Like HTML, XML elements have opening and closing tags (enclosed in brackets) that always have to match. Each element may have named attributes, with the attribute value specified in quotes. Elements may be nested to arbitrary levels. Each element may also have text content that is not included within brackets. Finally, if an element has no text or child elements, it may be abbreviated with an empty-element tag $(<$ Element $/>)$. As far as my use of XML goes, this is all the structure that is necessary, and this basic syntax is easy for students to acquire. There are also many XML editors with various niceties (e.g., syntax coloring, automatic formatting, well-formedness checks), but because XML files are merely text files, one can also use any garden-variety text editor or word processor.

XML also has a number of advantages that might prove useful in the future. For example, XML files can be associated with a document type definition (DTD) grammar that defines the possible arrangements of elements and attributes that can be used in a given XML application. Currently, there is no DTD for the present use of XML, but adding one could simplify the process of debugging scripts by ensuring that the XML file provides valid input. Another feature of XML is the ability to write transformation grammars that translate one form of XML to another. In principle, such transformations would allow one to write program scripts in an entirely different application (e.g., a graphical drawing program), export the document to XML, and then transform that output into a format that the experimental control program understands. Thus, using such an approach, one might be able to devise a graphical interface to the scripting environment.

The hierarchical structure in XML is important for the present application because experiments also have a hierarchical structure. For example, an experiment typically consists of a sequence of blocks, blocks consist of a sequence of trials, and trials consist of some arrangement of events. Furthermore, it is easy to conceptualize control flow within a trial as determined by nested events. For example, a stimulus presentation event might consist of a sequence of display events for a prime, a target, and a mask. A reaction time paradigm might include an event consisting of a race between a stimulus presentation event and a response event, with the superordinate event concluding when either of the child events finishes. (Moreover, the stimulus presentation event could be decomposed into a sequence of events as before.) Finally, a trial in a psychological refractory period paradigm might consist of the parallel presentation of two reaction time trials, with the superordinate event concluding only when both components have finished. Thus, flow control can be described by nested events that occur either in sequence or in parallel and parallel events that may complete exhaustively or in a race.

An XML representation can readily depict this hierarchical structure. For example, Table 2 shows an XML fragment that describes the hierarchical arrangement of blocks, trials, and events in an experiment. (An "id" attribute has been shown for each element to provide a name for each.) The essence of the hybrid approach I am describing is to use XML input such as this to construct a hierarchy of program objects. For example, each Block element would be used to generate an object in the Block class, each Trial element would be used to generate an object in the Trial class, and so on. The advantage of this approach is that it provides an environment that can be both easily scripted and easily extended. Little training and no programming skill is needed to acquire the basic XML formalism and to begin writing experimental control scripts, and students with a small amount of experience using the existing range of objects are able to develop relatively complex applications. Moreover, the system can be readily extended by programming new objects, which in turn can be used in subsequent scripts. Thus, the approach provides both the flexibility of specific-experiment programs and much of the efficiency of general-purpose programs.

\section{Some Details of ESExpt}

Although the ideas in the present hybrid approach can be implemented in many ways, at least some of the implementation details are crucial for allowing a broad range of experiments to be scripted with a minimal amount of effort. In the present section, I describe some of the details of my implementation using the Cocoa environment

Table 2

Experimental Structure in XMI

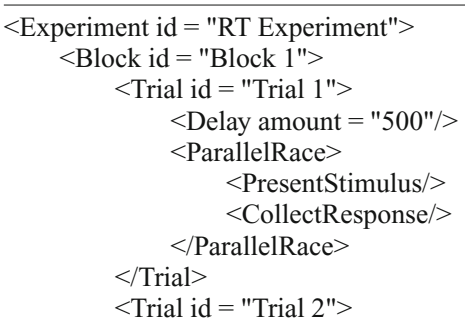


on Mac OS X. ${ }^{1}$ Cocoa is Apple's name for the collection of Objective-C APIs and runtime libraries for developing OS X applications (Apple Incorporated, 2007); Cocoa (as well as other programming tools) is provided free as part of OS X. A standard practice in that environment is to have two letters precede the name of object classes, to differentiate them from classes in other development projects. Objects in the present effort were preceded by ES for Experiment System, and the program that one runs is called ESExpt. I will discuss three interesting features: flow of control and event structure, parameters and parameter references, and replication blocks and lists.

Flow of control and event structure. Objects for running events, trials, and blocks are all descendants of an abstract class, ESExptElement, that embodies the basic mechanism for flow of control in ESExpt: Each element is started, starts its subordinate elements, and then, at an appropriate time, completes. Asynchronous messaging is used to communicate among elements at various levels. In particular, when an object is completed, it posts a message indicating its change in status. Its superordinate subsequently receives the message and performs an appropriate action. For example, when a trial completes, its superordinate block object will generally start the next trial or, if there are no more trials to be run, complete itself. Variations on this general scheme provide a mechanism for describing the flow of control during a trial with the hierarchical arrangement of different types of events. If the events are in sequence, the parent event initiates the next event in the sequence after each event is completed; if the events are to be done in a parallel race, all of the events are started at the same time and the parent is completed as soon as any of the children complete; and if the events are to be done exhaustively in parallel, the parent waits until all of the children have completed. There are also conditional events that take place only under a specified condition (e.g., to provide error feedback). This use of messaging allows event objects to be developed independently of the context in which they might appear. More generally, there is no need for any explicit description of the structure of an experiment other than the hierarchical arrangement of objects as described in the XML file.

Parameters and parameter references. Another feature that makes scripting a workable approach is that objects have parameters that define aspects of their behavior. In turn, parameters are objects themselves and are part of experiment hierarchy. This means that they are specified in the XML input file in exactly the same way as blocks, trials, and events. For example, Table 3 shows an event that delays an unspecified period of time. The duration of the delay is determined by an associated parameter with

Table 3

Parameters in the XML File

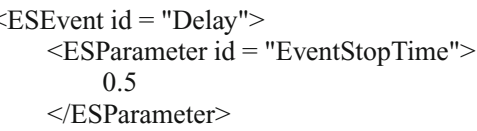

Table 4

Parameter References in the XML File

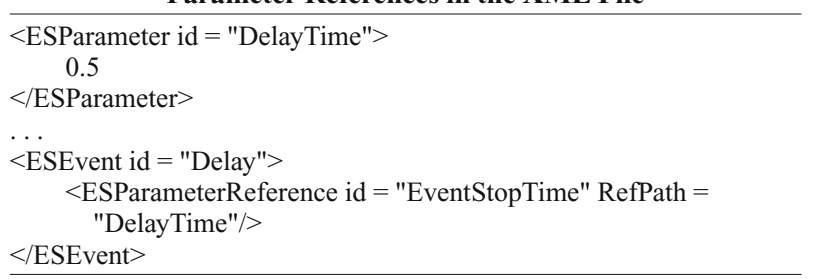

the id of "EventStopTime," which, in this case, indicates that the event should wait for $0.5 \mathrm{sec}$. As an XML shortcut, the ESExpt allows parameters to be specified as attributes rather than as nested elements. Thus, the excerpt in Table 3 could be expressed as

$$
<\text { ESEvent id = "Delay" EventStopTime = "0.5"/>. }
$$

Because parameters are commonly used in the experiment scripts, this makes the XML files much more compact and readable. Parameters are also used to describe the results of trials and events, with the nature of that output varying with the type of event. For example, all events will output duration, keypress events will additionally output the response key, and mouse-click events will output the click location.

Another key to developing expressive power is to provide for parameter references; a parameter reference is effectively a pointer to a parameter that can be found elsewhere in the experiment hierarchy. Table 4 illustrates the use of parameter references. Here, the delay duration is specified not as a parameter subordinate to the event element as before but via a reference to another parameter found previously in the XML file; the parameter to reference is specified by indicating its id attribute as a RefPath parameter. References allow the same parameter to be used by several elements and, critically, allow the output of one event to be used as the input to another. This enables, for example, a conditional event to test the output of a response event to assess whether error feedback should be provided.

Replication blocks and parameter lists. Another type of object is used to eliminate the need to explicitly list each of the experimental trials in the XML file. A replication block object replicates each child trial some number of times and optionally puts them in a random order. Thus, a replication block is effectively a type of macro that expands as the file is read in. Often, though, one does not want to merely duplicate a trial but, rather, to repeat it in different forms. For example, one might wish to use different stimuli on each trial, use different timing parameters, and so on. The solution here is the inclusion of parameter lists. A parameter list is a set of values, specified either in the XML input file or in a separate file, that are referenced systematically by the trials in a replication block. Table 5 provides an example of a replication block and a parameter list. At the beginning of the excerpt is a parameter list containing 20 text stimuli. Subsequently, a replication block is included that replicates a trial template 20 times. In the trial template, the text stimulus is identi- 
Table 5

Replication Blocks and Parameter Lists in the XML File

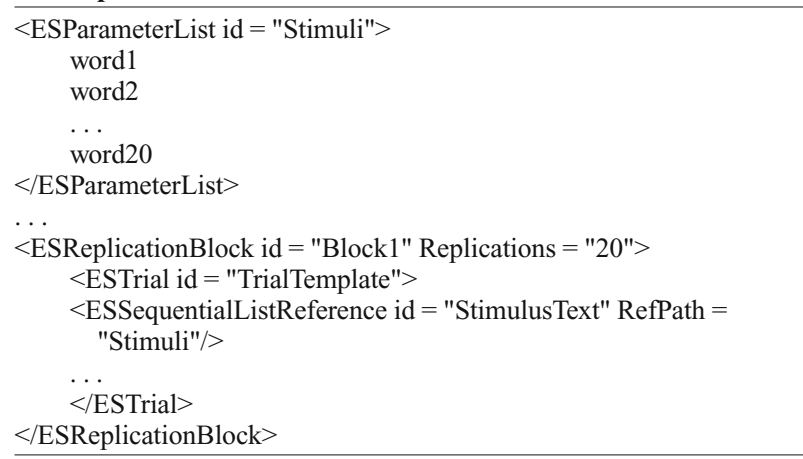

fied using a (sequential) list reference object. Each time the trial template is replicated, the list reference object is incremented so that it refers to successive items in the list. Consequently, each trial in the block will use a different stimulus. By coordinating several such lists, one can generate factorial designs in which different possible combinations of list values are represented in a block.

Other objects. A central advantage of the hybrid approach described here is that the contents of the XML "script" are entirely open ended; one can simply program more objects when one encounters a situation or problem that is not easily handled by the existing stable of objects. For example, at one point I wanted to be able to remotely monitor the status of an experimental session, so I created an ESLogEvent object that generates log messages on the OS X console; the experimenter can log on to the experimental machine (using ssh) and monitor these log messages using "tail" or similar commands. Over the last few years, the development of different types of event objects has been dictated by the stimulus/response requirements of various paradigms. This has led to events for presenting images, text, and sounds; events for mouse and keyboard responses; events for a digital I/O interface; and events for a software voice key and voice recognition. Such events span the bulk of dependent variables that are typically used in experimental psychology research. However, the programming to add objects that support more specialized requirements is straightforward. For example, I have developed events for recording input from the Ascension Technologies motion tracker.

A critical component of any experimental control program is output. ESExpt is a document-based application, and an ESExpt document stores the entire session context. This means that it can be saved and then reopened at a later time with no loss of information concerning the trials and data collected so far. For example, one could use such a document to collect data from a given subject across several experimental sessions. In addition, there are "summary" objects that allow one to export data to tab-delimited text files, which can then be imported by Excel or statistical analysis programs. One existing descendent of the summary object allows one to specify in the XML file which parameter values should be written to the output file. Moreover, one can specify different pa- rameter values to be exported from an ESExpt document even after the session has been completed. Of course, the value of the present approach is that new types of summary objects can be programmed, allowing flexibility to tailor the output capabilities of the system to the particular needs of a particular paradigm or analysis.

Extending ESExpt in this way requires knowledge of Objective-C, Cocoa, and the design of the core elements of the existing object hierarchy. Currently, ESExpt is extended by opening the project in Apple's programming environment, XCode, and defining a new object class. After the program is compiled, the new object would be automatically available to be included in any XML script. In keeping with the promise of object-oriented programming, modest changes and additions to ESExpt can often be accomplished with a minimum of coding and only a superficial appreciation of the overall program structure. For example, a summary object that computes summary statistics over a group of trials could be created by reimplementing a single method in an existing object class. This is because one merely needs to specify the new behavior for the class and can rely on the object to inherit the behavior of its ancestor object classes for the bulk of its functionality. On the other hand, there is no limit, in principle, to what such extensions to ESExpt can do. For example, if scripting trials in terms of individual events are deemed too cumbersome, one could write an object class that by itself implements all of the events of a trial for a particular paradigm. (This in fact is the use of the object-oriented programming advocated by Dixon [1991].)

\section{ESExpt in Action}

Tables 6, 7, and 8 provide examples of complete scripts that run demonstration experiments. Although these examples use a number of features of ESExpt that I have not discussed, they suffice to illustrate the level of complexity needed to write a script for a working experiment. Table 6 shows perhaps the simplest script that performs something useful: It presents two possible text stimuli ("X" and "O"), collects response time, and summarizes the response and its latency. Table 7 shows a somewhat more complicated reaction time paradigm that includes four possible image stimuli appearing in two possible locations on screen. Trials are subject initiated; error feedback is provided; log messages are generated so that errors can be monitored; and the stimulus, response, and response time are summarized for each trial. Table 8 shows a more conceptually demanding psychological refractory period paradigm. In this case, two tasks are run concurrently with potentially overlapping stimuli and responses. The program detects whether the responses are made in the correct order and provides feedback. These examples demonstrate that ESExpt scripts are much more concise than what would be needed in a general-purpose programming language. Although a certain amount of support may be necessary for students to develop a new paradigm from scratch, the scripts are fairly transparent, and repurposing existing scripts is straightforward and requires a minimum 
Table 6

Simple Reaction Time Example

$<$ ESExperiment id="TrivialExpt" $>$

$<$ ESSummary id="RT Summary" Title="RT Summary..."

SummaryParameters="Stimulus/Duration Stimulus/Response/ResponseKey" $/>$

$<$ ESParameterList id="Stimuli" $>$ X O $</$ ESParameterList $>$

$<$ ESReplicationBlock id="SingleBlock" Replications="8" Randomize="YES">

$<$ ESTrial id="Trial" $>$

$<$ ESEvent id="Delay" EventStopTime="0.5" >

$<$ ESTextEvent id="Stimulus" StimulusFontSize="24" >

$<$ ESSequentialListReference id="StimulusText" RefPath="Stimuli" $>>$

$<$ ESKeyboardResponseEvent id="Response" RespondingKeys="z/" /> $</$ ESTextEvent $>$

$<$ ESTrial $>$

$</$ ESReplicationBlock $>$

$</$ ESExperiment $>$

Table 7

More Elaborate Reaction Time Example

$<$ ESExperiment id="RTExpt" $>$

$<$ ESParameterSummary id="Summary" Title="RT Summary..." >

$<$ ESParameter id="SummaryParameters" $>$

StimulusResponse/Duration

StimulusResponse/ResponseEvent/ResponseKey

Correct

$</$ ESParameter $>$

$<$ ESParameterSummary $>$

$<$ ESReplicationBlock Replications="8" id="Block 1">

$<$ ESParameterList id="Files" $>$ BlurX.png CrystalX.png BlurO.png CrystalO.png

$<$ ESParameterList $>$

$<$ ESParameterList id="OffsetList" ParamListReps="4" ListDelimiters=",">

$-1000,1000$

$<$ /ESParameterList $>$

$<$ ESParameterList id="Responses" ParamListReps="2" > z $/<$ ESParameterList $>$

$<$ ESTrial id="XOTrial" $>$

$<$ ESIncrement id="TrialIndex" $>>$

$<$ ESListReference id="Correct" RefPath="Responses"

ListIndex="@TrialIndex" />

$<$ ESTextEvent id="Fixation" StimulusText="+" StimulusFontSize="24">

$<$ ESKeyboardResponseEvent id="ReadyEvent"

$</$ ESTextEvent $>$

RespondingKeys $=" \% 20 " />$

$<$ ESEvent id="Delay" EventStopTime="0.5" />

$<$ ESImageEvent id="StimulusResponse" $>$

$<$ ESListReference id="StimulusOffset" RefPath="OffsetList"

ListIndex="@TrialIndex" />

$<$ ESListReference id="ImageFileName" RefPath="Files"

ListIndex="@TrialIndex" $>$

$<$ ESKeyboardResponseEvent id="ResponseEvent"

RespondingKeys $=" \mathrm{z} / \mathrm{p} / \mathrm{>}$

$<$ /ESImageEvent $>$

$<$ ESConditionalEvent id="FeedbackEvent" Condition="ne" >

$<$ ESReference id="Parameter1" RefPath="Correct"/>

$<$ ESReference id="Parameter2"

RefPath="StimulusResponse/ResponseEvent/ResponseKey"/>

$<$ ESLogEvent id="Error Response" $>$

$<$ ESReference id="LogMessage" RefPath=

"StimulusResponse/ResponseEvent/ResponseKey" />

$<$ ESLogEvent $>$

$<$ ESTextEvent id="ErrorFeedback" EventStopTime="1.0"

StimulusFontName="Times"

StimulusFontSize="24"

StimulusText="Error"/>

$</$ ESConditionalEvent $>$

$</$ ESTrial $>$

$<$ ESReplicationBlock $>$

$</$ ESExperiment $>$ 
Table 8

Psychological Refractory Period Paradigm Example

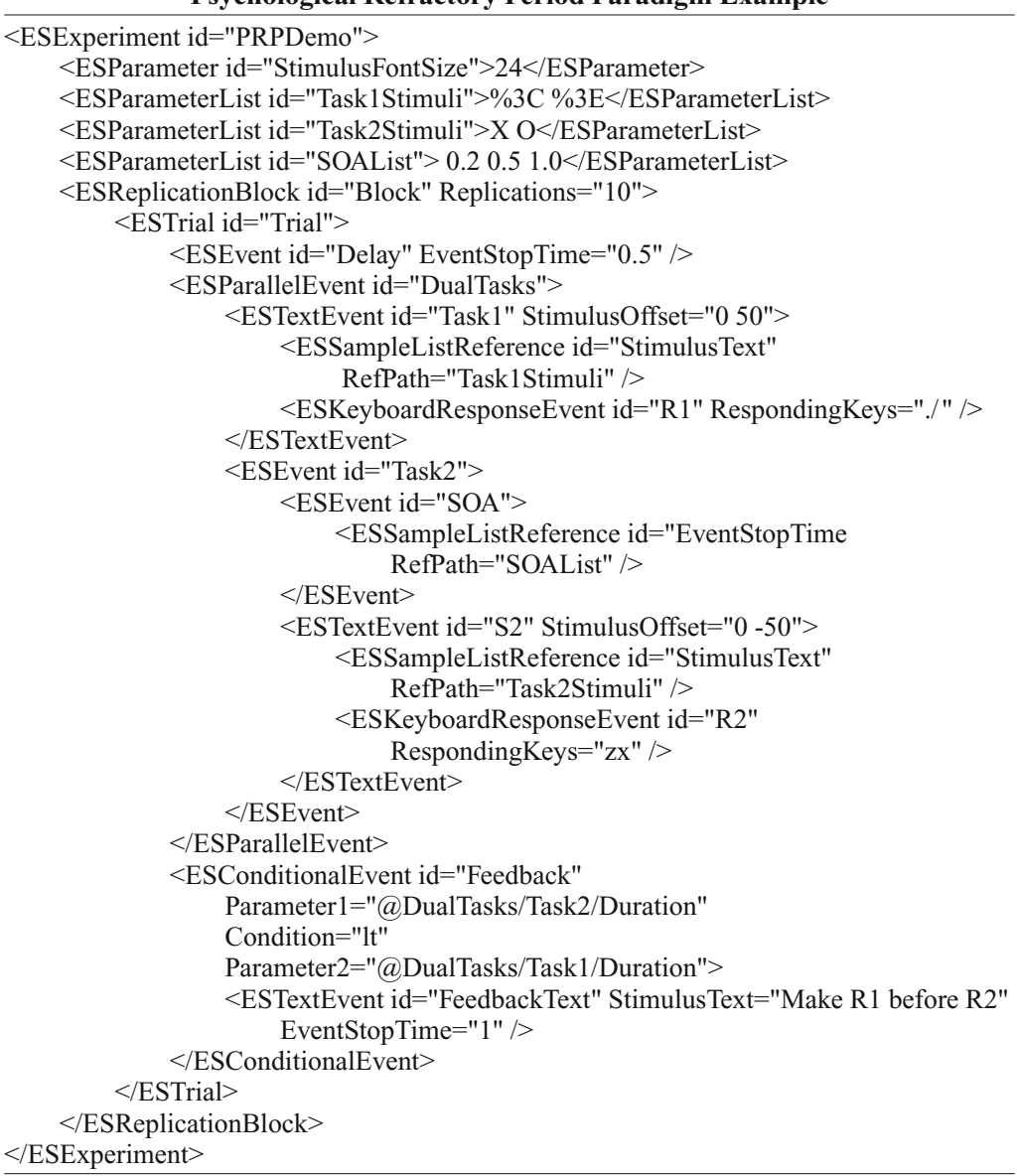

of training. A property of the XML input to ESExpt is that the input reflects not only a script for creating the objects to control the experiment, but also the structure of the program that results. In particular, while running, the program objects generally have precisely the same hierarchical structure as is apparent in the XML files, and this hierarchical structure embodies the entire logic of how the objects fit together to run an experiment. (An exception is the ESReplicationBlock, which expands to include the required number of instances of each trial.)

When an ESExpt document is opened, it is shown on the screen in an outline format that allows one to see minimally a summary of each trial. Figure 1 depicts the screen after ESExpt reads the XML file shown in Table 7. Before starting the experiment, one would normally hide the menu bar (using a provided menu command) and zoom the stimulus display panel to fill the entire screen. One can also interact with the outline view to interrogate the details of individual events. This is illustrated in Figure 2. In this case, the outline has been opened to examine the details of Trial 3, the "StimulusResponse" event has been opened, and, within that, the "ResponseEvent" event has been opened to reveal the event's duration and the collected response. There is also a limited capacity to edit the experimental hierarchy while the program is running. For example, one can add parameters or change their values. This feature is sometimes useful when piloting a paradigm, since one can change features such as timing or display characteristics without exiting the program.

Although no systematic assessment of the performance of ESExpt has been conducted, the program has built-in checks on timing constraints. For example, events that have a prescribed duration also store their actual duration as output parameters. Such indices indicate that the deviation from the nominal duration of events is generally no more than a millisecond or two using $1.8-\mathrm{GHz}$ PowerPC G5 iMacs in their normal configuration. Errors in timing reflect the time required to prepare events and move from one event or trial to the next, along with more general system overhead. Thus, timing is likely to be more precise with more current hardware and when concurrent system processes are eliminated. My experience is that paradigms that require a very large number of objects (such as might be needed to run thousands of trials in a session) may require more than the minimum memory configuration for adequate performance. In the present implementation, responses are collected using USB devices (e.g., the keyboard) that are polled at $60 \mathrm{~Hz}$, and there is no provision for synchronization with 


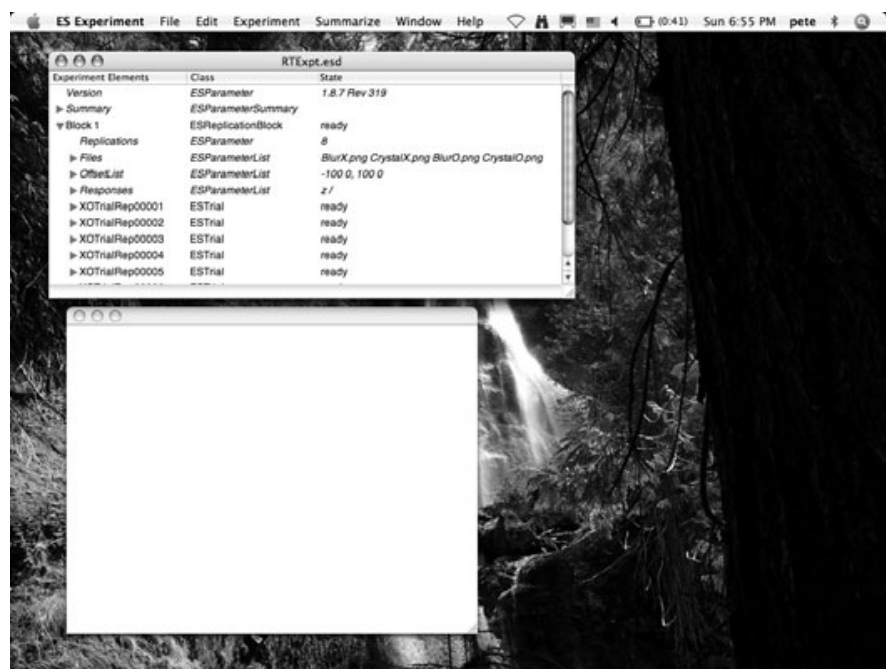

Figure 1. Appearance of ESExpt after opening the XML file in Table 7. The object hierarchy created to run the experiment is presented as an outline in the upper window, and stimuli will be presented in the lower blank panel.

the display synch signal. However, it would be straightforward to incorporate new objects to handle more precise $\mathrm{I} / \mathrm{O}$ timing within the present approach.

\section{Conclusion}

In sum, I have described a new way to address the flexibility/efficiency problem in writing experimental control programs. The approach involves writing objectoriented programs in which the program object hierarchy corresponds to the conceptual hierarchy in the experiment. Then, new experiments can be created by creating an XML script that names the objects to be used in the experiment and describes their hierarchical arrangement. A new experimental control program is then created by

\begin{tabular}{|c|c|c|}
\hline 000 & RTExpt.esd & \\
\hline Experiment Elements & Class & State \\
\hline Version & ESParameter & 1.8.7 $\operatorname{Rev} 319$ \\
\hline$\triangleright$ Summary & ESParameterSummary & \\
\hline Block 1 & ESReplicationBlock & paused \\
\hline Replications & ESParameter & 8 \\
\hline DFiles & ESParameterList & BlurX.png CrystalX.png BlurO.png Crysta/O.png \\
\hline$\checkmark$ Offsetlist & ESParameterList & $-1000,1000$ \\
\hline Desponses & ESParameterList & $z^{\prime}$ \\
\hline DOTrialRep00001 & ESTrial & completed \\
\hline DOTrialRep00002 & ESTrial & completed \\
\hline$\nabla$ XOTrialRep00003 & ESTrial & completed \\
\hline Triallndex & ESIncrement & 2 \\
\hline D Correct & ESListReference & / \\
\hline Duration & ESParameter & 5.748 \\
\hline Fixation & ESTextEvent & completed \\
\hline$\triangleright$ Delay & ESEvent & timedout \\
\hline$\nabla$ StimulusResponse & ESImageEvent & completed \\
\hline DStimulusoffset & ESListReference & -1000 \\
\hline DImageFileName & ESListReference & Bluro.png \\
\hline Duration & ESParameter & 3.476 \\
\hline VResponseEvent & ESKeyboardResponseEvent & completed \\
\hline RespondingKeys & ESParameter & $z^{\prime}$ \\
\hline Duration & ESParameter & 3.475 \\
\hline ResponseKey & ESParameter & I \\
\hline D FeedbackEvent & ESConditionalEvent & completed \\
\hline XOTrialRep00004 & ESTrial & halted \\
\hline XOTrialRep00005 & ESTrial & ready \\
\hline XOTrialRep00006 & ESTrial & ready \\
\hline 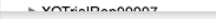 & resting & mand. \\
\hline
\end{tabular}

Figure 2. The appearance of the ESExpt document after running several trials with the outline opened to interrogate a particular event. dynamically creating the named objects in the indicated arrangement. Such an approach has many of the conveniences of a general-purpose program while maintaining the flexibility of a specific-experiment program.

\section{AUTHOR NOTE}

Correspondence concerning this article should be addressed to P. Dixon, Department of Psychology, University of Alberta, Edmonton, AB, T6G 2E9 Canada (e-mail: peter.dixon@ualberta.ca).

\section{REFERENCES}

APPle InCORPORATEd (2007). Cocoa. Retrieved April 23, 2008, from http://developer.apple.com/cocoa.

BonatTi, L. (2008, March). PsyScope X project. Retrieved April 23, 2008, from http://psy.ck.sissa.it.

CEDRus Corporation (2008). SuperLab home page. Retrieved April 23, 2008, from www.superlab.com.

Dixon, P. (1991). The promise of object-oriented programming. Behavior Research Methods, Instruments, \& Computers, 23, 134-141.

Dixon, P., \& Glover, S. (2008). The influence of relative size information on grasping: Evidence from a size-contrast illusion. Unpublished manuscript.

Dixon, P., \& Li, H. (2007, November). Mind wandering under dual task conditions. Poster presented at the Annual Meeting of the Psychonomic Society, Long Beach, CA.

Flatt, M., Cohen, J. D., \& Provost, J. (1994). PsyScope: User manual. Pittsburgh, PA: Carnegie Mellon University.

Harold, E. R., \& Means, W. S. (2004). XML in a nutshell: A desktop quick reference (3rd ed.). Sebastopol, CA: O'Reilly.

Psychology Software Tools, Inc. (n.d.). E-Prime. Retrieved April 23, 2008, from www.pstnet.com/products/e-prime.

Westbury, C. (2007, November). ACTUATE (Assessing Cases: The University of Alberta Testing Environment). Paper presented at the meeting of the Society for Computers in Psychology, Los Angeles.

\section{NOTE}

1. Binary and source code, examples, and (minimal) documentation are available from the author under the terms of the GNU General Public License.

(Manuscript received November 19, 2007; revision accepted for publication May 21, 2008.) 